\title{
Association of Fisher scale and changes of language in patients with aneurysmal subarachnoid hemorrhage
}

\author{
Associação da escala de Fisher com alterações da linguagem em pacientes com \\ hemorragia subaracnoide aneurismática
}

Moysés Loiola Ponte de Souza

\begin{abstract}
Resumo da Dissertação de Mestrado apresentada à Universidade Federal de Pernambuco. Área de Neurocirurgia. Recife PE, Brasil.
Correspondence: Moysés Loiola Ponte de Souza; Rua General Artur Oscar, 30 / ap. 1402; 52050-430 Recife PE, Brasil; E-mail: souzamoyses@hotmail.com Orientador: Hildo Rocha Cirne Azevedo Filho.
\end{abstract}

Received 25 June 2014; Received in final form 1 August 2014; Accepted 22 August 2014.

\begin{abstract}
Cognitive deficits caused by subarachnoid hemorrhage (SAH) after rupture of cerebral aneurysms are common, as approximately half of patients have severe, or at least striking, declines in one or more functions of the cognitive domain. The Fisher Scale is associated with the development of vasospasm and thus with the final performance of the patient after SAH. The association of this scale with language disorders in the period preceding the treatment has not been reported yet in the literature. Associate the presence of language deficits with varying degrees of the Fisher Scale in patients with SAH in the period preceding the treatment of aneurysm, as well as compare the various degrees of this scale, identifying the Fisher Scale degrees more associated with the decline of language. The database of 185 preoperative evaluations of language was studied, through the Montreal Toulouse Protocol Alpha version and verbal fluency through CERAD battery, of patients of Hospital da Restauração with aneurysmal SAH. The data relating to the Fisher Scale, the location of the aneurysm, the age and the gender of patients were obtained through review of medical records. Patients were divided according to the Fisher Scale (Fisher I, II, III or IV) and compared with a control group of individuals considered normal. Disorders in language and verbal fluency in patients with SAH in the preoperative period were evidenced. The classification of the patients according to the Fisher Scale allowed to identify differences between the sub-groups and to conclude that patients with bulkier bleeding (Fisher III and IV) have larger declines in the analyzed functions.
\end{abstract}

Keywords: Fisher scale, subarachnoid hemorrhage, aneurysm. 